\title{
Distinct behavioural profiles in frontotemporal dementia and semantic dementia
}

\author{
J S Snowden, D Bathgate, A Varma, A Blackshaw, Z C Gibbons, D Neary
}

\begin{abstract}
Objective-To test predictions that frontotemporal dementia and semantic dementia give rise to distinct patterns of behavioural change.

Methods-An informant based semistructured behavioural interview, covering the domains of basic and social emotions, social and personal behaviour, sensory behaviour, eating and oral behaviour, repetitive behaviours, rituals, and compulsions, was administered to carers of 41 patients with semantic dementia and with apathetic (FTD-A) and disinhibited (FTD-D) forms of frontotemporal dementia.
\end{abstract}

Results-Consistent with prediction, emotional changes differentiated FTD from semantic dementia. Whereas lack of emotional response was pervasive in FTD, it was more selective in semantic dementia, affecting particularly the capacity to show fear. Social avoidance occurred more often in FTD and social seeking in semantic dementia. Patients with FTD showed reduced response to pain, whereas patients with semantic dementia more often showed exaggerated reactions to sensory stimuli. Gluttony and indiscriminate eating were characteristic of FTD, whereas patients with semantic dementia were more likely to exhibit food fads. Hyperorality, involving inedible objects, was unrelated to gluttony, indicating different underlying mechanisms. Repetitive behaviours were common in both FTD and semantic dementia, but had a more compulsive quality in semantic dementia. Behavioural differences were greater between semantic dementia and FTD-A than FTD-D. A logistic regression analysis indicated that emotional and repetitive, compulsive behaviours discriminated FTD from semantic dementia with $97 \%$ accuracy.

Conclusion-The findings confirm predictions regarding behavioural differences in frontotemporal and semantic dementia and point to differential roles of the frontal and temporal lobes in affect, social functioning, eating, and compulsive behaviour.

(F Neurol Neurosurg Psychiatry 2001;70:323-332)

Keywords: frontotemporal dementia; semantic dementia; behaviour

Frontotemporal lobar degeneration is a primary cerebral atrophy associated with nonAlzheimer's pathology, estimated to account for $20 \%$ of cases of degenerative dementia with presenile onset. It gives rise to distinct clinical syndromes determined by the distribution of atrophy within the frontal and temporal lobes. Frontotemporal dementia refers to a disorder predominantly of behaviour. ${ }^{1-4}$ The most common behavioural changes are a loss of basic and social emotions, disinhibition, personal neglect, a generalised loss of interest in activities, overeating, altered preference for sweet foods, wandering, pacing, and motor and verbal stereotypies. In an earlier study (unpublished data) we found that changes in emotion and eating, together with behavioural stereotypies differentiated FTD from Alzheimer's disease and vascular dementia with $97 \%$ accuracy. Pathological examination disclosed atrophy in the frontal lobes and anterior temporal lobes. ${ }^{5-7}$

In FTD, two contrasting subsyndromes have been described ${ }^{2}$ : one of disinhibition, distractibility, and purposeless overactivity, associated with atrophy of the orbitomedial frontal lobes and temporal pole, the other of apathy, inertia, and loss of volition, occurring when the frontal lobe atrophy is widespread and extends into the dorsolateral frontal cortex. In both apathetic (FTD-A) and disinhibited (FTD-D) syndromes the marked behavioural disorder stands in contrast to the relative preservation of instrumental skills. Patients do not have primary aphasic symptomatology, or deficits in perceptual or spatial function.

Semantic dementia, by contrast, is a form of frontotemporal lobar degeneration in which deficits in language and visual perception represent the salient symptoms. ${ }^{9-11}$ Patients progressively lose conceptual knowledge about the world, affecting their ability to understand the meaning of words, visual percepts, and other sensory stimuli such as non-verbal sounds, tastes, and smells. ${ }^{8}{ }^{12}$ The syndrome is associated with circumscribed degeneration of the anterior temporal lobes, and both imaging $^{1314}$ and necropsy studies ${ }^{8}$ have shown the inferior and middle temporal gyri to be predominantly affected.

Although semantic deficits dominate the clinical picture, behavioural alterations also occur. Nevertheless our clinical findings have led to the suggestion that these are qualitatively different from those of FTD. ${ }^{8}$ Patients with semantic dementia, for example, characteristically show a narrowing of interests and often become preoccupied with a single activity such as doing jigsaws or painting, which they pursue assiduously. This contrasts with the general loss of interest and purposelessness of behaviour in FTD. Patients with semantic dementia have been noted to show hypochondriacal and histrionic traits, with overreaction to neutral sensory stimuli. This contrasts with the reduced reactivity of patients with FTD. Behaviour in 
patients with semantic dementia is time bound and seems to have a more compulsive quality than in FTD. Patients with semantic dementia show more emotional warmth and range of emotional expression than patients with FTD. Such differences are, however, presumptive, based on anecdotal reports by relatives and clinical observation of patients. Moreover, the only published study that has made a systematic comparison of behaviour in FTD and semantic dementia ${ }^{15}$ has elicited relatively few differences.

The purpose of the present study was to explore the nature of behavioural changes in FTD and semantic dementia in a new prospective patient cohort, involving blind investigators. A primary aim was to test out predictions about behavioural differences between the two, in the realm of affect and social, sensory, eating, and repetitive behaviours. It was predicted that (1) patients with FTD would show more pervasive loss of basic emotions than patients with semantic dementia; (2) patients with FTD would show greater disinhibition and social breakdown and more generalised loss of interest in activities; (3) patients with FTD would show reduced reaction to sensory stimuli, whereas patients with semantic dementia would show heightened reaction; (4) patients with FTD would be less discriminating in their eating habits, and patients with semantic dementia more discriminating; (5) repetitive behaviours would constitute simple motor mannerisms and stereotypies in FTD, and complex behavioural routines in semantic dementia; (6) compulsive traits would be more prevalent in semantic dementia.

It was suspected that the greatest behavioural differences would be elicited between semantic dementia and the apathetic form of FTD (FTD-A), because these two syndromes most clearly reflect the contrast between temporal and frontal lobe pathology. In patients with the disinhibited form of FTD (FTD-D), in whom frontal pathology is confined to the orbitomedial parts of the frontal lobes, and there is invariably involvement of the temporal pole, behavioural differences would be anticipated to be less clear cut. Indeed, the intimate anatomical connectivity between orbitofrontal and temporal cortex suggests that these two regions may share close functional links.

Behavioural comparisons between the subsyndromes of frontotemporal lobar degeneration ought to help to characterise these syndromes more fully. Moreover, they have the potential, by virtue of the syndromes' differential involvement of frontal and temporal lobes, to improve understanding of the functions of the frontal and temporal lobes and their contributions to behaviour.

\section{Methods}

PATIENTS

The patients were consecutive, new referrals to a specialist clinic for dementia with a clinical diagnosis of frontotemporal dementia $(n=30)$ or semantic dementia $(n=11)$. The diagnosis was based on historical information, neurological examination, and neuropsychological as- sessment and supported by findings on structural (magnetic resonance) and functional (SPECT) imaging. Patients in whom the clinical diagnosis was equivocal were excluded from the study. Patients with a progressive nonfluent aphasia were also excluded because they were too few $(n=2)$ to enable meaningful comparisons with other groups.

Patients with FTD exhibited profound breakdown in personality and social conduct, in the context of physical wellbeing. Neurological signs were absent or limited to primitive reflexes or mild akinesia and rigidity. The presence or absence of striatal signs was recorded in all cases. Neuropsychological investigation disclosed a frontal lobe syndrome in the absence of visuospatial impairment. Primary aphasia, as defined by the presence of paraphasias and grammatical errors in speech, was absent. However, speech output was reduced in quantity in some patients, commensurate with their adynamic state. Brain imaging showed atrophy and impaired function of the frontal regions. All patients fulfilled criteria for prototypical FTD. ${ }^{16}{ }^{17}$ Patients were separated on clinical grounds into those showing a predominantly disinhibited, distractible picture (FTD-D) $(n=18)$ and those showing an apathetic, inert pattern (FTD-A) $(\mathrm{n}=12)$ as previously described. ${ }^{28}$ A SPECT image representative of the two subgroups is shown in fig $1 \mathrm{~A}$ and $\mathrm{B}$.

Patients with semantic dementia presented with impairments in semantic memory, characterised by problems in word comprehension and naming, and face and object recognition. Neurological examination was normal or limited to mild akinesia and rigidity. The presence or absence of striatal signs was recorded in all cases. Structural imaging showed atrophy, most marked in the temporal lobes and SPECT imaging disclosed characteristic changes in the temporal regions. All patients fulfilled clinical criteria for semantic dementia. ${ }^{17}$ Illustrative SPECT is shown in fig $1 \mathrm{C}$.

Demographic details of patients at the time of the questionnaire are shown in table 1 . The FTD groups comprised an equal number of male and female patients, whereas there were more female patients in the semantic dementia group. There was a trend towards an older age of onset in patients with FTD-A compared with patients with FTD-D $(t=2.0, \mathrm{p}=0.06)$, but no age differences were present between the FTD groups and the semantic dementia group. The groups were well matched for duration of illness. Patients in the the FTD-A group, whose test performance is characterised by economy of effort and “don't know" responses, unsurprisingly performed worse on the mini mental state examination (MMSE) ${ }^{18}$ than both the FTD-D $(t=3.4, \mathrm{p}=0.002)$ and semantic dementia ( $t=3.8, \mathrm{p}=0.001)$ groups.

\section{INFORMANT BASED BEHAVIOURAL INTERVIEW}

Evaluation of behaviour was carried out using a semistructured, informant based interview, designed to examine behavioural changes in FTD. The full interview, described in relation to a comparative behavioural study of FTD, Alzheimer's disease, and cerebrovascular 
dementia (unpublished data) has been shown to yield good interrater reliability. The study evaluated the following aspects of behaviour.
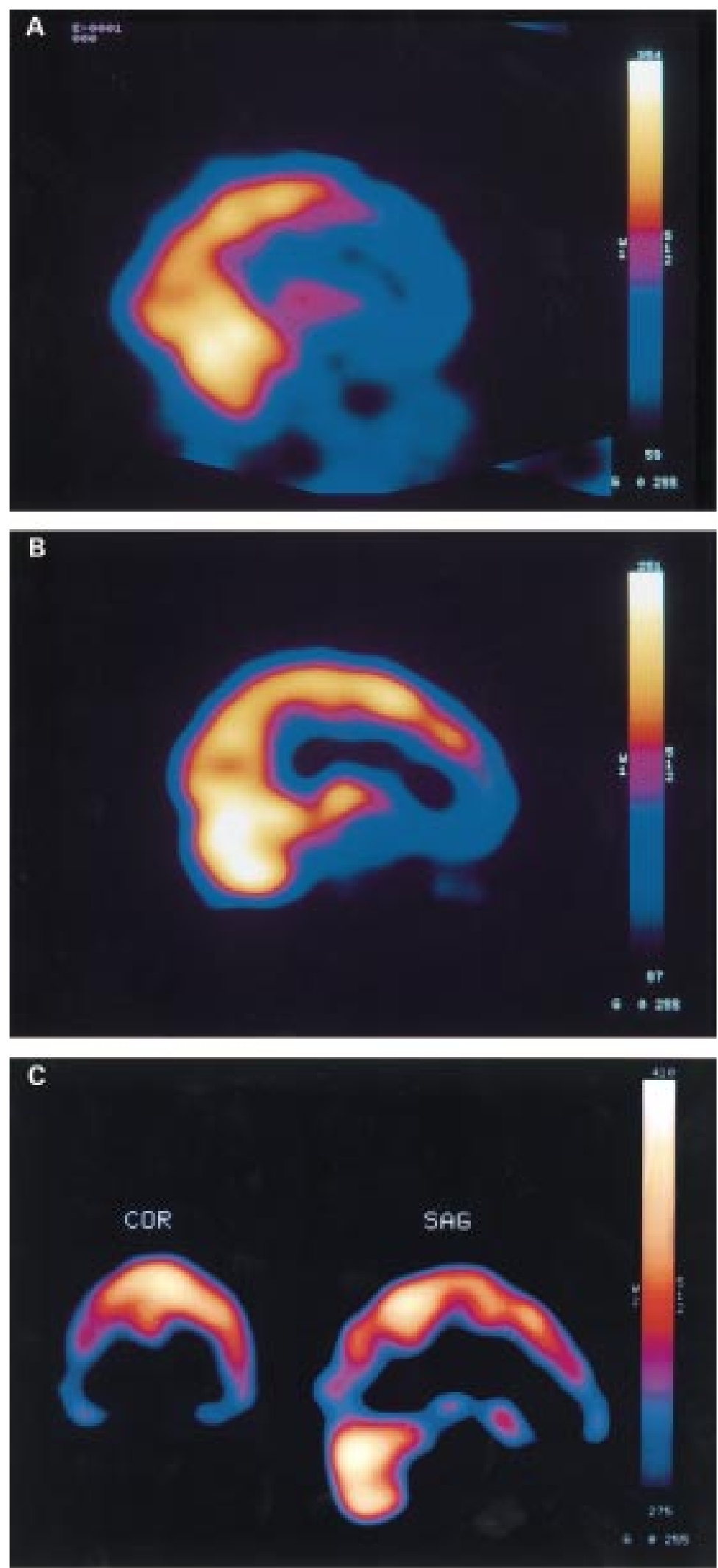

Figure 1 Sagittal view of SPECT in (A) an apathetic patient with FTD (FTD-A), showing widespread frontal lobe dysfunction, $(B)$ a disinhibited patient with FTD (FTD-D) showing frontal lobe dysfunction in orbital regions, and (C) a patient with semantic dementia. The coronal view in this patient demonstrates the reduced tracer uptake in the temporal lobes, particularly on the right side.
Table 1 Patient details

\begin{tabular}{llll}
\hline & FTD $A$ & FTD-D & Semantic dementia \\
\hline Number & 12 & 18 & 11 \\
Men & 6 & 9 & 4 \\
Women & 6 & 9 & 7 \\
Age at onset & $61(8)$ & $54(10)$ & $58(7)$ \\
Illness duration (y) & $4(1)$ & $4(3)$ & $5(3)$ \\
MMSE & $10(8)$ & $21(9)$ & $21(6)$
\end{tabular}

FTD-A=Apathetic presentation of FTD; FTD-D=disinhibited presentation of FTD. Values in parentheses are SD.

(1) Affect: basic emotions (the capacity to show happiness, sadness, anger, fear, surprise, disgust), social emotions (selfishness, loss of embarrassment), irritability, aggression, emotional insight (distress or concern when confronted by functional disabilities).

(2) Social and personal: disinhibition, social interactions (avoidance of social contact, seeks out social contact), self care, interest, and involvement in activities.

(3) Sensory: reaction to pain, heat, and cold.

(4) Eating and oral behaviours: overeating, cramming, altered food preference, food fads, mouthing of inedible objects.

(5) Repetitive behaviours, compulsions, and rituals: simple motor stereotypies (humming, hand rubbing, foot tapping), complex repetitive behavioural routines and rituals, verbal stereotypies and repetitive themes, wandering and pacing, environmentally dependent behaviours (hoarding, touching and counting objects, reading notices aloud, echolalia, echopraxia), and compulsive symptoms. The compulsive symptoms incorporated symptoms relating to order and routine, concern with cleanliness and repetitive checking, and paralleled items from the Leyton inventory for obsessive-compulsive disorder. ${ }^{19}$

(6) Cognitively mediated behaviours: use of wrong words, failure of object recognition, mutism.

PROCEDURE

Interviews were conducted with the primary carer, normally spouse, or partner. It was emphasised that a "symptom" should reflect a substantive change from the patient's premorbid state and not a longstanding character trait. At the beginning of the interview the informant was asked to recall the onset of the illness to permit demarcation of the period of illness for which questions applied. Great care was taken by the interviewer to ensure that each symptom being probed was understood. The same illustrative examples were given at each interview, ensuring consistency of presentation. Responses for each question were coded as 1 or 0 , representing the presence or absence of notable change from the premorbid state.

The interviewer had no clinical contact with the patient, either before or at the time of the interview, was unaware of the patient's group classification, and was blind to the specific hypotheses under investigation.

ANALYSIS

Data analyses were carried out using the SPSS-PC software package. Items of the Behavioural Interview were analysed individually using $\chi^{2}$ tests, with post hoc pairwise Fisher's 
Table 2. Frequency of behavioural symptoms in apathetic (FTD-A) and disinhibited (FTD-D) forms of FTD and semantic dementia

\begin{tabular}{|c|c|c|c|c|c|c|c|}
\hline & \multicolumn{3}{|c|}{ Frequency (\%) } & \multirow[b]{2}{*}{$\begin{array}{l}\text { Overall } \\
\chi^{2}\end{array}$} & \multicolumn{3}{|c|}{ Individual group comparisons (Fisher's exact test) } \\
\hline & $F T D-A$ & $F T D-D$ & $S D$ & & $\begin{array}{l}F T D-A \text { v FTD-D } \\
\text { p Value }\end{array}$ & $\begin{array}{l}F T D-D v S D \\
p \text { Value }\end{array}$ & $\begin{array}{l}F T D-A v S D \\
p \text { Value }\end{array}$ \\
\hline \multicolumn{8}{|l|}{ Affect: } \\
\hline Loss of basic emotions & 100 & 83 & 60 & 6.1 & NS & NS & 0.03 \\
\hline Exaggerated emotional display & 33 & 39 & 55 & 1.1 & & & \\
\hline Irritability & 50 & 61 & 82 & 2.6 & & & \\
\hline Aggression & 25 & 61 & 64 & 4.7 & 0.06 & NS & 0.07 \\
\hline Loss of emotional insight & 75 & 78 & 9 & 15.2 & NS & 0.000 & 0.002 \\
\hline Loss of embarrassment & 92 & 89 & 73 & 3.5 & & & \\
\hline Selfishness & 83 & 89 & 91 & 0.3 & & & \\
\hline \multicolumn{8}{|l|}{ Social and personal behaviour: } \\
\hline Disinhibition & 58 & 61 & 36 & 1.6 & & & \\
\hline Social avoidance & 50 & 17 & 18 & 4.6 & 0.06 & NS & NS \\
\hline Seeks out social contact & 17 & 28 & 72 & 8.8 & NS & 0.02 & 0.01 \\
\hline Neglect of hygiene & 92 & 83 & 64 & 3.0 & & & \\
\hline General loss of interest & 91 & 89 & 18 & 19.4 & NS & 0.000 & 0.001 \\
\hline \multicolumn{8}{|l|}{ Sensory behaviours: } \\
\hline Loss of awareness of pain & 45 & 39 & 27 & 0.8 & & & \\
\hline Exaggerated reaction to pain & 0 & 33 & 55 & 8.5 & 0.03 & NS & 0.005 \\
\hline Exaggerated heat/cold response & 0 & 11 & 45 & 9.2 & NS & 0.05 & 0.01 \\
\hline \multicolumn{8}{|l|}{ Eating and oral behaviours: } \\
\hline Overeats/gluttony & 83 & 61 & 36 & 5.3 & NS & NS & 0.03 \\
\hline Crams food & 50 & 50 & 9 & 5.7 & NS & 0.03 & 0.04 \\
\hline Less selective/indiscriminate & 55 & 41 & 9 & 5.3 & NS & 0.08 & 0.03 \\
\hline Eats continually if food present & 50 & 41 & 27 & 1.3 & & & \\
\hline Seeks out food & 17 & 44 & 18 & 3.6 & & & \\
\hline Preference for sweet foods & 25 & 56 & 36 & 2.9 & & & \\
\hline Preference for savoury foods & 0 & 0 & 9 & 2.8 & & & \\
\hline More selective/food fads & 8 & 22 & 55 & 6.6 & NS & 0.09 & 0.02 \\
\hline \multirow{2}{*}{\multicolumn{8}{|c|}{ Repetitive behaviours, compulsions, and rituals: }} \\
\hline & & & & & & & \\
\hline \multicolumn{8}{|c|}{ Behavioural stereotypies } \\
\hline Simple motor stereotypies & 75 & 44 & 55 & 2.7 & & & \\
\hline Complex routines & 8 & 39 & 55 & 5.8 & 0.07 & NS & 0.02 \\
\hline Verbal stereotypies & 25 & 67 & 73 & 6.8 & 0.06 & NS & 0.03 \\
\hline Repetitive themes & 33 & 28 & 91 & 12.1 & NS & 0.001 & 0.007 \\
\hline Wandering & 83 & 56 & 18 & 9.8 & NS & 0.05 & 0.003 \\
\hline Pacing & 58 & 61 & 36 & 1.8 & & & \\
\hline Paces fixed route & 33 & 33 & 0 & 4.8 & NS & 0.04 & 0.06 \\
\hline \multicolumn{8}{|l|}{ Environmentally dependent behaviours: } \\
\hline Aligns objects & 50 & 28 & 18 & 2.9 & & & \\
\hline Hoards objects & 42 & 67 & 64 & 2.0 & & & \\
\hline Touches or handles objects & 33 & 44 & 45 & 0.5 & & & \\
\hline Counts objects & 8 & 17 & 9 & 0.6 & & & \\
\hline Reads notices aloud & 25 & 33 & 55 & 1.5 & & & \\
\hline Copies speech (echolalia) & 33 & 33 & 18 & 0.9 & & & \\
\hline Imitates actions (echopraxia) & 0 & 9 & 18 & 3.3 & & & \\
\hline \multicolumn{8}{|l|}{ Compulsive behaviours: } \\
\hline Clockwatching & 8 & 39 & 82 & 12.9 & 0.07 & 0.03 & 0.001 \\
\hline Adherence to daily routine & 8 & 17 & 73 & 14.0 & NS & 0.004 & 0.002 \\
\hline Does tasks same way & 25 & 28 & 45 & 1.3 & & & \\
\hline Completes tasks in same order & 8 & 22 & 36 & 2.6 & & & \\
\hline Arranges belongings in same way & 8 & 6 & 55 & 11.8 & NS & 0.006 & 0.02 \\
\hline Performs tasks again and again & 17 & 17 & 55 & 5.9 & NS & 0.04 & 0.07 \\
\hline Upset if routine disrupted & 17 & 33 & 45 & 2.2 & & & \\
\hline Excessive worrying & 17 & 11 & 64 & 10.5 & NS & 0.005 & 0.0 \\
\hline Needs to do things immediately & 36 & 44 & 64 & 1.8 & & & \\
\hline Preoccupied checking locks etc & 17 & 22 & 9 & 0.8 & & & \\
\hline Checks and rechecks what done & 8 & 17 & 27 & 1.5 & & & \\
\hline Excessive attention to detail & 8 & 22 & 36 & 2.6 & & & \\
\hline Overconcern with cleanliness & 8 & 0 & 18 & 3.4 & & & \\
\hline Superstitious rituals & 22 & 17 & 9 & 0.8 & & & \\
\hline \multicolumn{8}{|l|}{ Cognitively mediated behaviours: } \\
\hline Fails to recognise objects & 17 & 17 & 55 & 5.9 & NS & 0.05 & 0.07 \\
\hline Uses wrong words & 36 & 17 & 91 & 15.6 & NS & 0.000 & 0.01 \\
\hline Mutism & 42 & 11 & 0 & 7.8 & 0.07 & NS & 0.02 \\
\hline
\end{tabular}

FTD-A=Apathetic presentation of FTD; FTD-D=disinhibited presentation of FTD; SD=semantic dementia. Prevalence figures greater than $50 \%$ are printed in bold.

exact tests for those items yielding a significant overall group effect. Mann-Whitney tests were used to compare the number of compulsive features reported in each of the groups. Two tailed tests were adopted. Logistic regression analyses were carried out to determine the degree to which behavioural characteristics separated FTD from semantic dementia.

\section{Results}

Table 2 shows the frequency of behavioural changes for FTD-A, FTD-D, and semantic dementia. Frequencies refer to the percentage of the sample in whom the behavioural abnormality was reported to be present, an abnormality being defined as a notable change from the patient's premorbid state. Pairwise group comparisons (Fisher's exact test) are shown only for those behavioural features that yielded an overall group effect in the three group $\chi^{2}$ analysis.

PRIMARY EMOTIONS

Alterations in emotions were prominent features of all three groups. A reduced capacity to show basic emotions was universally reported 
in FTD-A, but also commonly reported in FTD-D and semantic dementia. Only the FTD-A versus semantic dementia comparison reached statistical significance. However, an exploration of specific basic emotional states (happiness, sadness, fear, anger, surprise, and disgust), disclosed different patterns of findings. The greatest group differences occurred for sadness, anger, and disgust. That is, most patients with semantic dementia continued to show those emotions, whereas patients with FTD-A did not. Seventy three per cent of patients with FTD-A were reported to lack the capacity to show sadness, compared with only $27 \%$ of patients with semantic dementia (Fisher's exact test $\mathrm{p}=0.04) ; 75 \%$ of patients with FTD-A failed to show anger, compared with $27 \%$ of patients with semantic dementia (Fisher's exact test $p=0.03) ; 92 \%$ of patients with FTD-A no longer showed disgust, compared with only $27 \%$ of patients with semantic dementia (Fisher's exact test, $p=0.002$ ). Frequencies for patients with FTD-D were intermediate between those for patients with FTD-A and those with semantic dementia: $56 \%$ for sadness, $44 \%$ for anger, and $59 \%$ for disgust. The basic emotion most commonly lost in semantic dementia was fear. Loss of fear was reported in $55 \%$ of patients with semantic dementia compared with $67 \%$ of patients with FTD-A and those with FTD-D. Group differences were non-significant.

Change in primary emotions was characterised in FTD largely by a reduction in emotional expression, whereas in semantic dementia exaggerated emotional displays were reported as often as diminished emotional response. Whereas no patients with FTD-A or FTD-D were reported to show an increase in their demonstration of surprise this was reported in $36 \%$ of patients with semantic dementia (Fisher's exact test, $p=0.04$ and $p=0.01$ for the FTD-A and FTD-D comparison respectively).

Irritability and aggression were numerically more common in semantic dementia and FTD-D than FTD-A, although differences did not reach significance.

Patients with semantic dementia showed greater emotional insightfulness, as defined by their demonstration of distress, anxiety, or concern when confronted by their difficulties. Differences were highly significant.

SOCIAL EMOTIONS

Selfishness, selfcentredness, and lack of empathy or concern for others were virtually ubiquitous in all groups. Also shared by all groups was the loss of feelings of embarrassment. No group differences emerged.

SOCIAL BEHAVIOUR

Socially disinhibited behaviour was reported more often in patients with FTD than in those with semantic dementia, although this difference did not reach significance. Statistical group differences did emerge for social interactions. Apathetic patients (FTD-A) were more likely actively to avoid social contact, whereas patients with semantic dementia were more likely actively to seek it out.
INTEREST AND MOTIVATION

The capacity to maintain interest in activities strongly differentiated the groups. A generalised loss of interest in activities was almost invariably reported in patients with FTD, whereas it was rare in semantic dementia. Personal neglect and a need for prompting to attend to personal hygiene were reported commonly in all groups. Although numerically more frequent in FTD groups than in the semantic dementia group, differences did not reach significance.

\section{SENSORY BEHAVIOURS}

Loss of awareness of pain was reported numerically more often in FTD than semantic dementia, although group differences did not reach significance. The converse, an exaggerated reaction to tactile stimuli, showed the opposite pattern. It was commonly reported in semantic dementia, sometimes in FTD-D, and never in FTD-A. The difference between FTD-A and semantic dementia was highly significant. Patients with semantic dementia were also significantly more likely than patients in the other groups to show an exaggerated reaction to heat and cold.

\section{EATING AND ORAL BEHAVIOURS}

Alterations in eating behaviour occurred in all groups. However, the nature of the change differed. Gluttony, food cramming, indiscriminate eating, and continued eating while food was present characterised FTD, whereas increased selectivity and food fads characterised semantic dementia. These differences were significant. Altered food preference was in all groups almost invariably in the direction of increased preference for sweet foods, reported most often in FTD-D. These disinhibited patients were also more commonly reported to seek out food. Oral exploration of inanimate objects was rare in all groups, although numerically more common in FTD-D and semantic dementia than FTD-A.

\section{REPETITIVE BEHAVIOURS}

Repetitive behaviours were reported in all groups. Simple motor stereotypies, such as grunting, humming, lip smacking, hand rubbing, or foot tapping, were reported most often in FTD-A (75\% of patients), but also commonly in other groups $(44 \%$ and $55 \%$ of patients), so that group differences were non-significant. By contrast, complex repetitive behavioural routines, such as the tendency to sing the same song or recite the same rhyme over and over again, to clap the same rhythm repetitively, or dance the same dance, occurred significantly more often in FTD-D and semantic dementia than in FTD-A. Exploration of these complex behaviours (not shown in the table), indicated that clapping the same rhythm was the greatest single discriminator, being reported in $0 \%$ of patients with FTD-A, 33\% of those with FTD-D, and $45 \%$ of those with semantic dementia (Fisher's exact test: FTD-A $v$ FTD-D $\mathrm{p}=0.03$; FTD-A $v$ semantic dementia $\mathrm{p}=0.01$ ). In $22 \%$ of patients with FTD-D and $18 \%$ of patients with semantic dementia, 
but no patients with FTD-A complex routines were executed at fixed times each day. It was difficult for the carer to stop the routine being carried out without causing distress in $36 \%$ of patients with semantic dementia, but only $8 \%$ of those with FTD-A and 6\% of those with FTD-D.

Repetitive behaviours in the verbal domain were also discriminating. Verbal stereotypies, constituting repetition of a single word or phrase, were common in both FTD-D and semantic dementia and differentiated these groups from the FTD-A group. The preoccupation with and repetition of complete themes was, however, common only in semantic dementia and strongly differentiated semantic dementia from FTD.

Repeated wandering was significantly more common in FTD than semantic dementia. Pacing was also reported more often in FTD. Group differences for pacing only were nonsignificant. However, pacing a fixed route significantly distinguished both FTD groups from semantic dementia.

\section{ENVIRONMENTAL DEPENDENCY}

Some repetitive behaviours, such as echolalia, echopraxia, touching objects, continuing to eat when food remains available, and reading notices aloud are environmentally driven rather than internally driven. There was not a consistent relation between externally driven, stimulus bound behaviours, and subgroup type. Hoarding and the tendency to handle objects were reported more often in patients with FTD-D and those with semantic dementia than patients with FTD-A, and patients with semantic dementia were more commonly reported to read aloud public notices. However, echolalia was reported more often in FTD than semantic dementia. Echopraxia was rare in all groups. None of the group differences reached significance.

\section{COMPULSIONS AND RITUALS}

Behavioural symptoms relating to order and routine, paralleling items from the Leyton obsessive-compulsive disorder questionnaire, were summated to yield a compulsive symptom score. The median score was 0.5 for the FTD-A group, 2 for the FTD-D group, and 5 for the semantic dementia group. Individual Mann-Whitney tests showed that patients with semantic dementia had significantly more compulsive symptoms than both FTD-A $(z=2.5, \mathrm{p}=0.01)$ and patients with FTD-D $(z=2.1, \mathrm{p}=0.04)$. Patients with FTD-D had more compulsive symptoms than patients with FTD-A $(z=2.0, \mathrm{p}=0.05)$. Inspection of table 2 shows that compulsive symptoms in semantic dementia take the form predominantly of clockwatching tendencies and adherence to routine. Other symptoms traditionally associated with obsessive-compulsive disorder, such as repetitive checking of locks and lights switches, were reported only rarely.

COMPULSIVE SYMPTOMS AND STRIATAL SIGNS

Limb rigidity, reported by neurologists (DN and $\mathrm{AV}$ ) who were blind to the behavioural findings, was present in $42 \%$ of patients with FTD-A, 22\% of patients with FTD-D, and no patients with semantic dementia. The difference in frequency of occurrence of rigidity between the FTD-A and semantic dementia groups was significant (Fisher's exact test, $p=0.04)$. There was no association between the presence of striatal signs and the number of compulsive symptoms.

COGNITIVELY MEDIATED BEHAVIOURS

Consistent with their clinical classification, patients with semantic dementia were significantly more likely to fail to recognise objects and to use incorrect words than the FTD groups. By contrast, mutism was most common in patients with FTD-A and was reported in no patients with semantic dementia.

\section{LOGISTIC REGRESSION ANALYSIS: FTD VERSUS} SEMANTIC DEMENTIA

A stepwise logistic regression analysis was carried out. Initially, two variables alone entered the analysis and correctly classified $95 \%$ of the total group, $100 \%$ of patients with FTD, and $80 \%$ of patients with semantic dementia: loss of emotional insight and loss of the emotion of disgust (fig $2 \mathrm{~A}$ ). The presence of insight and the capability of showing disgust favoured semantic dementia, whereas loss of insight and an absence of feelings of disgust favoured FTD. These two variables continued to be most

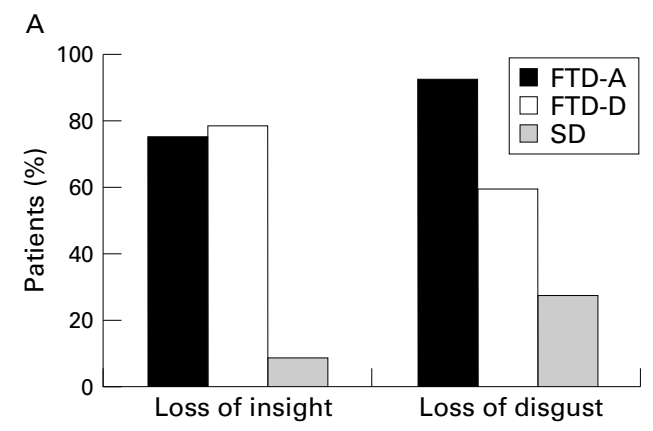

B

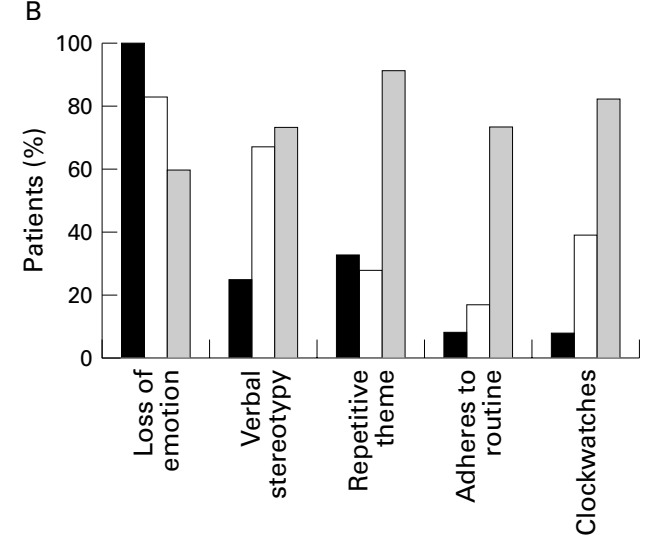

Figure 2 Behavioural features that best discriminate apathetic and disinhibited presentations of FTD from semantic dementia (SD). Regression analysis shows that loss of emotional insight and loss of disgust response $(A)$ classifies $95 \%$ of patients with FTD and those with semantic dementia. Loss of basic emotional responses, together with repetitive and compulsive behaviours (verbal stereotypies, repetitive themes, adherence to routine and clockwatching) (B) correctly classifies $97 \%$ of patients. 
discriminating when semantic dementia was compared with FTD-A alone and with FTD-D alone.

When these two variables were excluded a further five variables entered the analysis, and correctly classified $97 \%$ of the total group, $100 \%$ of patients with semantic dementia, and $96 \%$ of patients with FTD. These variables were loss of basic emotions, verbal stereotypies, repetitive themes, complex repetitive routines, and clockwatching (fig $2 \mathrm{~B}$ ). The loss of basic emotions favoured FTD whereas the presence of verbal stereotypies, repetitive conversational themes, repetitive behavioural routines, and clockwatching favoured semantic dementia.

Patients with FTD-D exhibited behaviours intermediate between those of FTD-A and semantic dementia. When these patients were omitted from the analysis, then the FTD-A group could be differentiated with 95\% accuracy from the semantic group on the basis of two variables only: loss of basic emotions and clockwatching. The first favoured FTD-A, the second semantic dementia. One hundred per cent of patients with semantic dementia were correctly classified and $91 \%$ of patients with FTD.

In a separate analysis of the two FTD subgroups, only the variable "verbal stereotypies" entered the analysis, allowing correct classification of $71 \%$ of patients with FTD-D and $82 \%$ of patients with FTD-A.

\section{Discussion}

The study shows several behavioural differences between FTD and semantic dementia, largely consistent with our clinical observations and predictions. In general, differences are greatest between semantic dementia and FTD-A, although significant differences also pertain with FTD-D.

The study shows that FTD is characterised by a general reduction in emotional expression, encompassing each of the basic emotions: happiness, sadness, anger, fear, disgust, and surprise. Patients exhibit a lack of insightfulness, as defined by their lack of emotional concern when their difficulties are exposed. Emotional blunting is most marked in FTD-A, but is present also in FTD-D. The findings in semantic dementia suggest a different pattern. Although there is reduction in emotional expression, this is not uniform across emotions. Typically, patients continue to show a range of emotions, which include anger, surprise, and disgust. They are emotionally insightful, and show distress at disability. The emotion most commonly reported to be lost is fear.

Selective loss of fear in some patients with semantic dementia is of interest in that it is one component of the Klüver-Bucy syndrome, described in monkeys with lesions of the anterior temporal lobes. ${ }^{20}$ We have previously drawn attention to the lack of awareness of danger in patients with semantic dementia ${ }^{8}$ and postulated that this might relate directly to the patients' semantic loss: patients no longer have conceptual knowledge about the potentially adverse properties of objects (for example, boiling water) or situations (for example, cross- ing a busy road). Whether semantic loss itself provides a sufficient explanation of patients' selective loss of fear is an open question. As the amygdala has been implicated in the expression of fear in both animals, ${ }^{21}{ }^{22}$ and humans ${ }^{23}{ }^{24}$ it remains possible that the loss of fear in these patients results from pathological changes in the amygdala. However, consistent with the semantic explanation is the finding that some patients with semantic dementia, but not those with FTD, show an increase in expression of surprise. That is, they are surprised to discover properties of an object (for example, that a glass breaks when dropped) that would normally be taken for granted. It would be reasonable to hypothesise that the temporal neocortex itself has a role in the expression of fear in permitting conceptual recognition of potentially threatening stimuli.

We have previously described patients with semantic dementia as having an increasingly narrowed, egocentric world view. In keeping with this, patients with semantic dementia are reported to be selfish, lacking in sympathy and empathy for others. However, whereas in FTD-A the loss of social emotions parallels a lack of interest in social interaction and social avoidance in many cases, most patients with semantic dementia continue to enjoy social interaction and are reported actively to seek it out. Thus, the groups differ qualitatively in their social functioning. Patients with FTD-A are asocial, whereas patients with semantic dementia are social, yet lack the capacity for empathic feeling necessary for successful social relationships. These findings are of contemporary relevance. Social behaviour in FTD has been the focus of recent studies ${ }^{25}$ and the temporal lobes have been ascribed a critical role. ${ }^{26}{ }^{27}$ The present findings support the view that the temporal lobes contribute to social behaviour, but emphasise that their role is not unique. Frontal lobe dysfunction has a more damaging and pervasive effect.

A generalised loss of interest in activities is a virtually ubiquitous feature of FTD, whereas it is rare in semantic dementia. This finding is consistent with our previous findings that patients with FTD, even those who are restless and overactive, exhibit a lack of purposeful goal directed behaviour, whereas patients with semantic dementia continue to pursue activities purposefully, albeit with excessive time expenditure on a narrowed repertoire of activities such as doing jigsaws or painting.

We have previously found that patients with FTD may show a loss of response to pain, and this is a highly differentiating feature from Alzheimer's disease. By contrast, we have noted that patients with semantic dementia may show an exaggerated reaction to sensory stimuli. ${ }^{8}$ The distinction is largely supported by this study, although with qualification. Although loss of pain response is present in a relatively high proportion of patients with FTD, it occurs too in some patients with semantic dementia. Moreover, some patients with FTD-D (but not patients with FTD-A) show exaggerated sensory responses. Reduced and exaggerated responses to sensory stimuli seem not to be 
mutually exclusive: a patient with semantic dementia with histrionic traits who reacts melodramatically to neutral sensations, such as light touch, may also fail to show an appropriate withdrawal reaction to painful stimuli. This suggests that exaggerated reactions are unlikely to reflect genuine hypersensitivity, along a pain threshold continuum, but rather may have a distinct underlying substrate.

Consistent with previous reports ${ }^{15} 28$ alterations in eating habits are a common feature, present in all groups. However, whereas gluttony, food cramming, and indiscriminate eating characterises FTD, particularly apathetic patients, patients with semantic dementia are typically more selective in their food intake. Mouthing of inanimate objects is rare in all groups, an unsurprising finding because it is typically a late feature. Of interest, however, is that attempts to eat non-food items are reported in patients with FTD-D and patients with semantic dementia, but not in those with FTD-A. The dissociation between overeating and hyperoral tendencies involving inedible objects suggests that the two involve different underlying mechanisms. Hyperorality is not a product of gluttony, and does not lie along an "overeating" continuum. It would be reasonable to suppose that, like fear, this feature of the Klüver-Bucy syndrome may in part be related to impaired semantic knowledge: patients are no longer able to differentiate conceptually between edible and non-edible items and so explore items orally. An altered preference for sweet foods occurs in a proportion of patients in all groups. Nevertheless, its numerically greater frequency in FTD-D and semantic dementia than in apathetic FTD, again suggests that this feature is not a general product of increased gluttony.

Our previous observations of patients with FTD and semantic dementia led to the prediction that patterns of repetitive behaviour would differ along the dimension of complexity. ${ }^{8}$ We suspected that frontal lobe dysfunction involving the dorsolateral cortex would lead to elementary repetitive behaviours and perseverations, such as grunting, humming, or hand rubbing, whereas orbitofrontal and particularly temporal lobe dysfunction would be associated with more elaborate repetitive behavioural routines. The findings are largely in accord with the prediction, although there is not a clear cut double dissociation. In FTD-A there is, as predicted, a marked tendency towards simple repetitive behaviours and virtual absence of complex repetitive routines. However, patients with semantic dementia and patients with FTD-D show simple behaviours as well as complex repetitive behaviours. Complex routines take various forms. For example, one patient with FTD-D, on entering a room, would tap out the same rhythm on each of the four walls before being seated. Another sang Ave Maria repetitively. Another counted every step made with his left foot and multiplied this by two every 100 steps to announce how far he had walked.

Repetitive behaviours in the verbal domain differentiate the three groups, and the dimension of complexity again seems relevant.
Repeated use of a single word or phrase is common in both FTD-D and semantic dementia, but not FTD-A. By contrast, repetition of an entire conversational theme (with virtually identical wording on each occasion) is a prominent feature only in semantic dementia.

Obsessive-compulsive symptoms have often been reported in FTD, ${ }^{29-33}$ and may represent a presenting symptom. ${ }^{33}$ Their presence seems paradoxical, in view of the fact that damage to the frontal lobes results in reduced anxiety and concern, and lesioning of the frontal lobes has been used as a surgical treatment of obsessivecompulsive disorder. The findings of the present study suggest that it is patients with semantic dementia, in whom temporal rather than frontal lobe pathology is prominent, who show most compulsive symptoms. Functional imaging studies of patients with idiopathic obsessive-compulsive disorder have disclosed hyperactivity of the orbital frontal cortex relative to normal. ${ }^{34}{ }^{35}$ It might be speculated that in semantic dementia the imbalance between poorly functioning temporal lobes and normally functioning frontal lobes results in a similar state of relative frontal overactivity.

Compulsive symptoms in patients with semantic dementia are characterised predominantly by clockwatching tendencies, rigid adherence to a fixed routine, carrying out tasks in precisely the same way, and preoccupation with a limited range of topics leading to repetitive conversational themes. Symptoms do not commonly have the quality of a superstitious ritual. Moreover, patients do not typically show prototypical obsessive-compulsive disorder symptoms such as checking and rechecking of locks and light switches, and they may exhibit a reduced rather than increased level of concern for personal appearance and hygiene.

Patients' semantic loss might potentially contribute to the pattern of compulsive symptoms. Patients have a progressively shrinking world view, with concepts increasingly limited to patients' daily experience. Numerical concepts, including those pertaining to time, however, remain relatively well preserved. Temporally bound routines might help to provide a meaningful framework to patients' lives which would otherwise be absent. Irrespective of their underlying basis, the circumscribed nature of patients' compulsive symptoms reinforces the view that distinct symptom profiles of obsessive-compulsive disorder may exist. ${ }^{36}$ In keeping with this notion, despite documented evidence for an association between symptoms of obsessive-compulsive disorder and striatal pathology, ${ }^{37-39}$ there was no association in this study between compulsive behaviours pertaining to order and routine and the presence of striatal signs on neurological examination. By contrast, our previous experience of patients with FTD suggests that overtly superstitious and ritualistic behaviours, such as avoiding walking on cracks between paving stones, are most likely to occur in patients with FTD with marked striatal pathology. ${ }^{8}$ It may be that both temporal lobe and striatal dysfunction contribute to obsessive-compulsive disorder-type behaviours but that they do so in different ways. 
Not surprisingly, cognitively mediated behaviours of failure of object recognition and use of wrong words occur significantly more often in semantic dementia than in FTD. Of note, however, is that mutism is more likely in FTD-A, indicating a dissociation between aphasic symptomatology and mutism. The finding reinforces the notion that mutism is a reflection of patients' general inertia, unresponsiveness, and adynamia and not a product of a primary aphasia.

Significance values shown in this study are uncorrected. In view of the small numbers in the groups it was expected that significance values might be small and that there was a danger of making type 2 statistical errors if an excessively conservative approach was adopted, particularly as the variables, although numerous, are not totally independent. Conservative two tailed rather than one tailed tests were, nevertheless, adopted, despite predictions of differences in one direction. The strength of the differences between FTD and semantic dementia, the consistency in the pattern of differences across related variables, and the concordance of the findings with previous prediction suggest that group differences are real and not a result of chance.

This study shows prominent differences between FTD and semantic dementia in the domains of emotion, social, sensory, eating, and stereotyped behaviours. At first sight these findings seem to contradict those of Bozeat et $a l,{ }^{15}$ who demonstrated relatively few differences. Nevertheless, there are differences between the studies. The primary intention of the study by Bozeat et al was to compare FTD with Alzheimer's disease, so that the domains of behaviour covered by their questionnaire were inevitably broad. The present study showed that it is qualitative characteristics of those broad behavioural domains where group differences lie. Moreover, the domain of emotion, an important discriminator in the present study, was not explicitly addressed by the questionnaire of Bozeat et al. Interestingly, however, inspection of their data discloses numerically greater clockwatching behaviour in semantic dementia than "frontal-variant" FTD and more overeating behaviour in FTD, suggesting some parallels with the present study.

A comment is warranted about differences in use of terminology. We use the term frontotemporal dementia here to apply exclusively to the behavioural disorder described in the LundManchester consensus statement, ${ }^{16}$ whereas Bozeat and colleagues adopt it as an umbrella term, which includes both the behavioural disorder and clinical syndrome of semantic dementia. The term frontotemporal dementia, as a designation for the behavioural syndrome, was adopted by the Lund-Manchester groups in favour of "dementia of frontal type" or "frontal lobe dementia" in recognition of the finding, based on examination of 100 brains, that the behavioural disorder is invariably associated with atrophy of the temporal poles as well as the frontal lobes. Temporal lobe atrophy may be present even in patients whose disease course is attenuated by the cooccurrence of motor neuron disease $^{8}$ suggesting that it is a relatively early feature. In patients with the disinhibited form of FTD, pathological changes in the orbitofrontal cortex and temporal pole seem to be inextricably linked, so that neither a designation of "frontal variant" or "temporal varian" FTD is entirely appropriate. These factors suggest a need for caution in the automatic attribution of behavioural change to frontal lobe pathology, as implied by the term "frontal variant FTD". It is by comparing clinical syndromes, associated with overlapping distributions of pathology (widespread frontal+temporal pole $v$ orbitofrontal+temporal pole $v$ anterior temporal) that the potential role of these different brain regions can be better judged. Features of Kluver-Bucy syndrome, for example, have been regarded as consistent with frontal variant FTD,${ }^{15}$ yet the present study would suggest a closer association with temporal than frontal lobe pathology.

Behavioural differences in the present study ought to reflect predominantly differential roles of the frontal and temporal lobes. Although morphological studies ${ }^{5-8}$ indicate that the degenerative process in FTD and semantic dementia may involve structures such as the thalamus, amygdala, and hippocampus, damage is often mild and would seem to occur relatively late in the disease course, as those structures are relatively spared in patients whose disease course is attenuated by the presence of motor neuron disease. ${ }^{8} 40$

In the present study the poorer performance on the mini mental state examination in the FTD-A group raises the question whether behavioural differences are a product of severity of illness. There are arguments against such an account. Firstly, economy of mental effort and "don't know" responses in FTD-A invariably yield test scores that underestimate patients' actual capabilities. Scores do not, therefore, provide a reliable estimate of severity. Secondly, patients with FTD-A are well matched to other groups for duration of illness. Thirdly, group differences are not always in the direction of more frequent symptoms in patients with FTD-A. The presence of behavioural dissociations suggests that severity itself is unlikely to be a sufficient explanation. Fourthly, differences are not limited to the FTD-A group. Robust group differences are present between semantic dementia and FTD-D, well matched both for duration of illness and MMSE scores. Fifthly, and most crucially, group differences are based on the presence or absence of behavioural symptoms occurring at any stage of the illness, not merely the present time. It is of interest in this regard that, by contrast with the large differences with semantic dementia, relatively minor differences emerge between FTD-A and FTD-D. This may be because some patients with FTD-A are disinhibited in the very early part of their illness, a feature that points to the orbitofrontal cortex as an early site of pathology in FTD.

Patients with FTD conform to current clinical criteria, ${ }^{17}$ which place weight on behavioural change. The issue of logical circularity 
therefore arises: are group differences merely a reflection of the fact that they are thus defined in the first place? Several factors argue that this is not so. Firstly, semantic dementia is defined largely on cognitive grounds, so that the presence or absence of behavioural changes similar or dissimilar to those of FTD was, at the outset, an open question. Secondly, behavioural changes are not invariably in the direction of higher frequency in FTD than semantic dementia: there are dissociations in behavioural pattern across the groups. Thirdly, diagnostic criteria for FTD identify only broad domains of behavioural change, such as altered eating habits and behavioural stereotypies. The present study highlights qualitative group differences within those domains, such as gluttony versus food fads and simple mannerisms versus complex repetitive routines. Finally, behavioural differences include features, such as exaggerated response to sensory stimuli, which are not included in current consensus criteria.

Divisions between clinical syndromes are not absolute. Clinical overlap would inevitably have the effect of reducing behavioural differences between groups. The fact that strong behavioural differences can nevertheless be identified, which accords with previous clinical experience, suggests that such differences are real and robust. The study draws attention to behavioural distinctions between the clinical syndromes of FTD and semantic dementia in the domains of emotion and insight, social behaviour, response to sensory stimuli, eating and oral behaviour, and repetitive behaviours and compulsions. The logistic regression analysis highlighted two principle areas of function that are particularly discriminating: emotion and repetitive-compulsive behaviours. Patients with FTD show a generalised reduction in emotional response, whereas in semantic dementia emotional changes are more specific. Repetitive behaviours in semantic dementia can be distinguished from those in FTD along the dimensions of complexity and compulsivity. Patients with semantic dementia are more likely to establish repetitive behavioural routines and to clockwatch. The findings emphasise the critical importance of the frontal lobes in mediating emotions, and point to temporal lobe dysfunction as an important determinant of compulsive behaviour. Future correlative studies of behavioural change and structural and functional change on brain imaging would provide further understanding of the anatomical substrate of the behavioural disorders of frontotemporal lobar degeneration.

1 Gustafson L. Frontal lobe degeneration of non-Alzheimer type. II. Clinical picture and differential diagnosis. Archives type. II. Clinical picture and differential diagno

2 of Gerontology and Geriatrics 1987;6:209-23. lobe type. $\mathcal{F}$ Neurol Neurosurg Psychiatry 1988;51:353-61. 3 Miller BL, Cummings JL, Villanueva-Meyer J, et al. Frontal lobe degeneration: clinical, neuropsychological
SPECT characteristics. Neurology 1991;41:1374-82. SPECT characteristics. Neurology 1991;41:1374-82. Gustafson L. Clinical picture of frontal lobe deg

non-Alzheimer type. Dementia 1993;4:143-8.
Brun A. Frontal lobe degeneration of non-Alzheimer type. I.

Neuropathology. Archives of Gerontology and Geriatrics 1987;6:193-208.
6 Mann DMA, South PW. The topographic distribution of brain atrophy in frontal lobe dementia. Acta Neuropathol brain atrophy in frontal

7 Mann DMA, South PW, Snowden JS, et al. Dementia of frontal lobe type; neuropathology and immunohistochemistry. F Neurol Neurosurg Psychiatry 1993;56:605-14.

8 Snowden JS, Neary D, Mann DMA. Frontotemporal lobar degeneration: frontotemporal dementia, progressive aphasia, semantic dementia. New York: Churchill Livingstone, 1996.

9 Snowden JS, Goulding PJ, Neary D. Semantic dementia: a form of circumscribed cerebral atrophy. Behav Neurol 1989;2:167-82.

10 Hodges JR, Patterson K, Oxbury S, et al. Semantic demenia. Progressive fluent aphasia with temporal lobe atrophy. Brain 1992;115:1783-806.

11 Snowden JS. Semantic dementia. In: O'Brien J, Ames D, Burns A, eds. Dementia. London: Edward Arnold, 2000:769-78.

12 Bozeat S, Lambon Ralph MA, Patterson K, et al. Non-verbal semantic impairment in semantic dementia. Neuropsychologia 2000;38:1207-15.

13 Mummery CJ, Patterson K, Wise RSJ, et al. Disrupted temporal connections in semantic dementia. Brain 1999;122: 61-73.

14 Mummery CJ, Patterson K, Price CJ, et al. A voxel-based morphometry study of semantic dementia: relationship between temporal lobe atrophy and semantic memory. Ann Neurol 2000;47:36-45.

15 Bozeat S, Gregory CA, Lambon Ralph MA, et al. Which neuropsychiatric and behavioural features distinguish frontal and temporal variants of frontotemporal dementia from Alzheimer's disease? ₹ Neurol Neurosurg Psychiatry 2000;69: 178-86.

16 The Lund and Manchester groups. Clinical and neuropathological criteria for frontotemporal dementia. $\mathcal{F ~ N e u -}$ rol Neurosurg Psychiatry 1994;57:416-18.

17 Neary D, Snowden JS, Gustafson L, et al. Frontotemporal lobar degeneration: a consensus on clinical diagnostic lobar degeneration: a consensus on
criteria. Neurology 1998;51:1546-54.

18 Folstein MF, Folstein SE, McHugh PR. Mini-mental state. f Psychiatr Res 1975;12:189-98.

19 Cooper J. The Leyton obsessional inventory. Psychol Med 1970;1:48-64.

20 Klüver H, Bucy PC. Preliminary analysis of functions of the temporal lobes in monkeys. Arch Neurol Psychiatry 1939;42:547-54

21 LeDoux JE. Emotion and the amygdala. In: Aggleton JP, ed. The amygdala. New York: Wiley-Liss, 1992, 339-51

22 Le Doux JE. Emotion: clues from the brain. Annu Rev Psychol 1995;46:209-35.

23 Adolphs R, Tranel D, Damasio H, et al. Fear and the human amygdala. F Neurosci 1995;15:5879-91.

24 Young AW, Aggleton JP, Hellawell DJ, et al. Face processing impairments after amygdalotomy. Brain 1995;118:15-24.

25 Miller BL, Darby A, Benson DF, et al. Aggressive, socially disruptive and antisocial behaviour associated with frontodisruptive and antisocial behaviour associated with fr
temporal dementia. Br f Psychiatry 1997;170:150-4.

26 Edwards-Lee T, Miller BL, Benson DF, et al. The temporal lobe variant of frontotemporal dementia. Brain 1997;120: lobe variant

27 Miller BL, Hou C, Goldberg M, et al. Anterior temporal lobes: social brain. In: Miller BL, Cummings JL, eds. The human frontal lobes: functions and disorders. New York: The Guilford Press, 1999:557-67.

28 Miller BL, Darby AL, Swartz JR, et al. Dietary changes, compulsions and sexual behavior in frontotemporal degeneration. Dementia 1995;6:195-9.

29 Ames D, Cummings JL, Wirshing WC, et al. Repetitive and compulsive behavior in frontal lobe degenerations. $\mathcal{F}$ Neuropsychiatry Clin Neurosci 1994;6:100-13.

30 Lawrence RM, Ronca MA, Tyrrell P, et al. Psychiatric symptoms in patients with focal cortical degeneration. Behav Neurol 1994;7:153-8.

31 Tonkology JM, Smith TW, Barreira PJ. Obsessivecompulsive disorders in Pick's disease. $f$ Neuropsychiatry Clin Neurosci 1994;6:176-80.

32 Stip E. Compulsive disorder and acquired antisocial behaviour in frontal lobe dementia. $\mathcal{F}$ Neuropsychiatry Clin Neuroci 1995; 7:116.

33 Mendez MF, Perryman KM, Miller BL, et al. Compulsive behaviors as presenting symptoms of frontotempora dementia. F Geriatr Psychiatry Neurol 1997;10:154-7

34 Baxter LR, Phelps ME, Mazziotta JC, et al. Local cerebral glucose metabolic rates in obsessive-compulsive disorder. Arch Gen Psychiatry 1987;44:211-8.

35 Baxter LR, Schwartz JM, Guze BH, et al. PET imaging in obsessive-compulsive disorder with and without depression. F Clin Psychiatry 1990;51:61-9.

36 Leckman JF, Grice DE, Boardman J, et al. Symptoms of obsessive-compulsive disorder. Am F Psychiatry 1997;154: 911-7.

37 Lopez-Rodriguez F, Gunay I, Glaser N. Obsessive compulsive disorder in a woman with left basal ganglia infarct: a sive disorder in a woman with left basal gan
case report. Behav Neurol 1997;10:101-3.

38 Berthier ML, Kulisevsky J, Gironell A, et al. ObsessiveBerthier ML, Kulisevsky J, Gironell A, et al. Obsessive-
compulsive disorder associated with brain lesions: clinical phenomenology, cognitive function, and anatomic correlates. Neurology 1996;47:353-61.

39 McGuire PK. The brain in obsessive-compulsive disorder. $\mathcal{F}$ Neurol Neurosurg Psychiatry 1995;59:457-9.

0 Neary D, Snowden JS, Mann DMA, et al. Frontal lobe dementia and motor neurone disease. $\mathcal{F}$ Neurol Neurosurg Psychiatry 1990;53:23-32. 